\title{
Questes
}

vestes Revue pluridisciplinaire d'études médiévales

Violences médiévales

\section{La violence du Perlesvaus : un défi à la critique?}

\section{Patrick Moran}

\section{OpenEdition \\ Journals}

\section{Édition électronique}

URL : http://journals.openedition.org/questes/363

DOI : 10.4000/questes.363

ISSN : 2109-9472

\section{Éditeur}

Les Amis de Questes

\section{Édition imprimée}

Date de publication : 15 mars 2008

Pagination : 8-21

ISSN : 2102-7188

\section{Référence électronique}

Patrick Moran, «La violence du Perlesvaus : un défi à la critique? », Questes [En ligne], 14 | 2008, mis en ligne le 01 janvier 2014, consulté le 19 avril 2019. URL : http://journals.openedition.org/ questes/363 ; DOI : 10.4000/questes.363 


\section{La violence du Perlesvaus : un défi à la critique?}

\section{Patrick MORAN}

Aux yeux des spécialistes de littérature arthurienne, le Perlesvaus, ou Haut livre du Graal comme l'appellent les manuscrits ${ }^{1}$, est une œuvre problématique par excellence. Roman en prose de la première moitié du XIII ${ }^{e}$ siècle, il survit dans neuf manuscrits, dont deux seulement sont complets ${ }^{2}$. L'intrigue qu'il déroule est une sorte de continuation du Conte du graal, mais avec des divergences importantes; le roman de Chrétien de Troyes est plus un point de départ pour le rédacteur qu'une véritable base pour son récit. Celui-ci se concentre sur trois chevaliers arthuriens, Perlesvaus (Perceval), Lancelot et Gauvain, et sur les aventures que la quête du Graal va leur faire vivre. Mais la quête telle qu'elle se présente dans le Perlesvaus ne ressemble pas beaucoup à la version diffusée par Le Conte du graal ou La Queste del saint Graal; autour d'elle se jouent moins le salut individuel des quêteurs et l'accomplissement mystique de leur destinée, que la survie d'un monde chrétien encerclé de toutes parts par les forces du mal et du paganisme. La quête du Graal devient progressivement une guerre du Graal, où l'ombre et la lumière s'affrontent sur un plan aussi bien physique que moral.

\footnotetext{
${ }^{1}$ C'est aussi le titre qu'a choisi Armand Strubel pour son édition récente : Le Haut livre du Graal, Armand Strubel (éd. et trad.), Paris, Livre de Poche, 2007. C'est de cette édition que je tirerai mes citations (en l'abrégeant en $H L G$ ). L'édition de référence du Perlesvaus demeure celle de William Nitze, Le Haut livre du Graal : Perlesvaus, William A. NitzE et T. Atkinson JENKINS (éds), Chicago, The University of Chicago Press, 1932-1937, 2 vol.

${ }^{2}$ OXFORD, Bodleian Library, Hatton 82 (sur lequel se fonde Nitze) et BRUXELLES, ms. 11145, Bibliothèque Royale des Ducs de Bourgogne. A. Strubel se fonde en revanche sur le manuscrit incomplet PARIS, BN fr. 1428, dont seuls le début et la fin manquent.
} 
Le Perlesvaus intrigue la critique pour de nombreuses raisons, dont la moindre n'est pas la question de sa datation : il est pour l'instant impossible de situer la composition du roman dans une fourchette plus précise que 1200-1250 environ, ce qui pose problème pour l'étude de l'émergence de la forme-prose : s'agit-il d'un des tous premiers exemples de prose romanesque, ou au contraire d'un surgeon tardif? Le contenu et le ton de ce roman ne sont pas pour rien dans cette difficulté : le Perlesvaus est une œuvre très éloignée de la "vulgate » arthurienne du début du XIII ${ }^{\mathrm{e}}$ siècle, aussi bien dans les événements qu'il décrit que dans l'idéologie qu'il manifeste, et on voit mal si ces divergences sont l'effet d'un rejet ou d'une ignorance des œuvres plus canoniques de l'époque.

L'aspect le plus déroutant est sans doute l'extrême violence manifestée par ce roman, violence qui aux yeux des modernes s'apparente souvent à de la cruauté : le Perlesvaus est rempli de décapitations, de massacres, d'exécutions sommaires, de supplices cruels et autres scènes sanglantes. Depuis la parution de l'édition Nitze, la critique cherche à justifier, expliquer ou comprendre cette violence, qu'elle décrit souvent en des termes très forts. Christine MarchelloNizia, dans sa traduction de $1989^{3}$, parle de l'«étrange férocité » de ce roman ; Charles Méla décrit l'action du Perlesvaus comme « un intervalle de violence entre le péché originel et l'eschatologie $»^{4}$, tandis que Jeanne Lods l'appelle un « conte barbare $»^{5}$; Nitze, dans son édition, parle de la «beauté sauvage et inhabituelle» ${ }^{6}$ de ce texte.

La violence à l'œuvre dans le Perlesvaus choque parce qu'elle est inhabituelle dans le contexte générique qu'est la matière de Bretagne : elle pose davantage problème que celle des chansons de geste, à laquelle elle est parfois

\footnotetext{
${ }^{3}$ Traduction d'extraits dans La Légende arthurienne. Le Graal et La Table Ronde, Danielle REGNIER-BOHLER (éd.), Paris, Robert Laffont, 1989, p. 119-309.

${ }^{4}$ Charles MÉla, La Reine et le Graal, Paris, Éditions du Seuil, 1984, p. 194.

5 Jeanne ODS, "Symbolisme chrétien, tradition celtique et vérité psychologique dans les personnages féminins du Perlesvaus », Mélanges Le Gentil, Paris, SEDES, 1973, p. 505-522, citation p. 516.

${ }^{6}$ « Wild and unfamiliar beauty », op. cit., I, p. ix.
} 
comparable d'ailleurs, mais qui est «normale» dans le contexte de la matière épique. Elle choque également en raison du propos religieux qui informe le roman; la Queste a habitué les médiévistes à davantage de spiritualité lorsqu'il est question du Graal ; le Perlesvaus, lui, fait l'éloge des valeurs proprement guerrières du chevalier, ignorant la distinction opérée par la Queste entre « chevalerie terrienne » et «chevalerie celestielle», au profit d'une opposition simple et frontale entre chrétiens et païens, Nouvelle Loi et Ancienne Loi.

La critique a élaboré un grand nombre de stratégies pour s'expliquer cette violence et la formuler de manière intelligible, mais la violence du Perlesvaus est tous azimuts, kaléidoscopique, et les explications unitaires qui tentent de la réduire à un principe ou une source fonctionnent mal. Le problème n'est pas tant la violence elle-même que son surgissement dans des contextes inattendus, pour des raisons qui sont difficiles à cerner.

\section{La barbarie au royaume de Bretagne}

La littérature médiévale n'est pas étrangère à la violence ; que ce soit dans les chansons de geste, les fabliaux ou Le Roman de Renart, les scènes brutales ou cruelles ne sont pas épargnées aux lecteurs des XII ${ }^{\mathrm{e}}$ et XIII ${ }^{\mathrm{e}}$ siècles. Dans le cadre du roman arthurien, en revanche, elles sont plus rares. La violence n'est évidemment pas absente de ces textes, dans la mesure où ils racontent les exploits guerriers et aventureux de chevaliers experts au combat; mais la violence arthurienne est généralement policée et intégrée dans les limites tolérées par la logique courtoise. Structurellement, le Perlesvaus n'est pas très éloigné d'un roman breton «classique»: les aventures et les quêtes qui le composent sont de facture traditionnelle, et ses personnages principaux font partie du personnel habituel de cette littérature, qu'il s'agisse d'Arthur, de Guenièvre, de Gauvain, de Lancelot ou de Keu; on retrouve bon nombre de 
motifs arthuriens canoniques tels que le don contraignant, les tournois ou les banquets à la cour d'Arthur.

Si le fonctionnement du récit est clairement romanesque, il subit pourtant un gauchissement, un assombrissement tout particuliers. Les personnages n'exhibent pas la pitié qu'on attend habituellement de la part de chevaliers de la Table Ronde, comme à la fin de ce duel entre Perlesvaus et Aristor :

Perlesvaus senti le colp qui grant fu et son hiaume enbarré ; il revient vers Aristor si tres durement qu'il li enpaint son glaive tres par mi le cors et abat lui et le cheval tot en un mont; puis est descendus sor lui, si li oste la coife del hauberc et deslace la ventaille. "C'avez vos enpensé a faire? fait Aristor. - Je vos trencherai la teste, dist Perlesvaus, si le presenterai a ma seror, a qui vos avez failli ! - No ferez, fait Aristor, mais laissiez me vivre et je vos pardonrai ma haine. - De vostre haine me soufferai je bien, ce m'est avis, d'ore en avant, fait Perlesvaus, mais la vie ne poet plus demorer en vos, kar vos l'avez bien deservi, et Damnedieus ne vielt sofrir !» Il li trenche la teste tot errant et la pent a l'arçon de sa sele $[\ldots]$. $^{7}$

Un roman de facture plus classique aurait sans doute vu Perlesvaus épargner son adversaire et le forcer à se constituer prisonnier auprès d'Arthur. Perlesvaus n'est pas le seul personnage à manquer de pitié et de clémence : Lancelot fait pendre des brigands qui menaçaient un ermitage ${ }^{8}$, et plusieurs demoiselles au fil du roman réclament les têtes de leurs ennemis aux chevaliers errants qui acceptent de les aider. Cette habitude de tuer les adversaires au lieu de les épargner devient même un moteur narratif : chaque victime ayant des parents désireux de faire payer le meurtrier, le cycle de la vengeance relance perpétuellement le récit. Il est toutefois remarquable que cette dureté ne se limite pas aux personnages de second plan ou à un héros faillible tel que Lancelot, entaché par son adultère avec la reine; Perlesvaus lui-même, qui est à bien des

\footnotetext{
${ }^{7} H L G$, branche XI, p. 932.

${ }^{8} H L G$, branche VIII, p. 456.
} 
égards le repère moral du texte, est aussi peu enclin à la mansuétude que les autres.

D'autres éléments inhabituels noircissent le trait du Haut livre du Graal : il s'agit d'un des rares romans arthuriens à mettre en scène un suicide, celui du roi du Château Mortel $^{9}$, qui est certes un païen voué aux flammes de l'enfer, mais dont la mort frappe tout particulièrement l'imagination par son aspect délibéré et inattendu. Le roman abonde également en décapitations et en têtes coupées, que ce soient les têtes transportées par la charrette de la demoiselle chauve $^{10}$, celles qui ornent le château des griffons ${ }^{11}$, ou les nombreuses têtes pendues aux selles des chevaliers (comme on le voit dans l'exemple cité cidessus); ce ne sont pas que les têtes qui se font trancher d'ailleurs, les mutilations en tous genres abondent. Armand Strubel parle d' « obsession » ${ }^{12}$ de la part du rédacteur; en 1963 Roger Loomis allait jusqu'à employer le vocabulaire de la psychiatrie, en décrivant l'auteur du Perlesvaus comme un malade mental, dérangé, paranoïaque, voire légèrement schizophrène ${ }^{13}$.

Les châtiments cruels sont légion. Marin le Jaloux torture son épouse parce qu'il est convaincu qu'elle l'a trompé avec Gauvain :

Il [Marin] s'arme tot errant et fait amener son cheval, puis fait la dame despoillier en pure sa chemise, qui li crioit merchi en plorant molt douchement. Il monte sor son cheval et prent son escu et son glave, et fait le nain prendre la dame par les tresches et le fait mener aprés li en la forest, et areste desor un lac d'une fontaine et le fait entrer la u l'iauge

\footnotetext{
${ }^{9} H L G$, branche IX, p. 690.

${ }^{10} H L G$, branche II, p. 186.

${ }^{11} H L G$, branche X, p. 806.

${ }^{12} H L G$, Introduction, p. 59.

${ }^{13}$ Roger Sherman Loomis, The Grail, from Celtic Myth to Christian Symbol, Cardiff/New York, University of Wales Press/Columbia University Press, 1963, «Perlesvaus: Welsh Talismans and a Welsh Elysium », p. 97-134. Les arguments cliniques de Loomis ne portent pas uniquement sur la violence du roman et sont, à bien y regarder, contestables, résultant pour la plupart d'une confusion entre ce que le narrateur déclare et ce que l'auteur historique croyait réellement. Ils sont néanmoins le signe de l'incrédulité souvent exprimée par la critique face à ce roman singulier et déroutant, dont on est tenté d'attribuer les bizarreries à une forme d'idiosyncrasie.
} 
sordoit plus parfont et descent et quiolt grans verges et chinglans en la forest ; et le conmenche a batre et a ferir tres par mi le dos et les mameles, si que li ruissiaus de la fontaine estoit tos sanglans. ${ }^{14}$

Le motif du sang et de la violence mêlés à l'eau se retrouve à de nombreuses reprises dans le roman : ici, mais également lors du suicide du roi du Château Mortel, dont le corps tombe dans l'eau, ou lorsque Perlesvaus fait jeter dans un fleuve les cadavres de centaines de païens ${ }^{15}$. La cruauté n'est pas toujours infligée aux femmes, comme dans le cas de la femme de Marin le Jaloux; elles peuvent elles aussi se montrer cruelles. Nombreuses sont les demoiselles, on l'a dit, à réclamer les têtes de leurs adversaires; la maîtresse du Château des Barbes se fait servir ses repas par une cohorte de chevaliers mutilés de diverses façons ${ }^{16}$; la Demoiselle Orgueilleuse a construit trois pièges habiles destinés à décapiter Lancelot, Gauvain et Perlesvaus ${ }^{17}$.

La cruauté et la violence semblent omniprésentes dans le roman, et aucun personnage, aucune instance n'y échappe : même les chevaliers les plus nobles exhibent une dureté peu commune. Cette noirceur, on l'a dit, surprend dans un roman breton. Si le Perlesvaus présente une version sombre de l'univers arthurien, force est de reconnaître qu'il est le seul roman de sa période à aller aussi loin. Plusieurs critiques modernes, gênés par cette «incohérence» générique, ont tenté de la détourner ou de la gommer. Pour Jean-Charles Payen, «c'est un roman épique, qui flatte le vieux rêve de l'Occident chrétien, tel que l'exprime la chanson de geste. ${ }^{18} \mathrm{Ce}$ serait donc une sorte d'hapax, un roman qui serait aussi une épopée - ce qui ne résout pas vraiment le problème. Au niveau de ses structures, de son personnel et de sa thématique, le Perlesvaus n'a rien de l'épopée et tout du roman, ce qui rend sa tonalité d'autant plus

\footnotetext{
${ }^{14} H L G$, branche IV, p. 244.

${ }^{15} H L G$, branche IX, p. 666.

${ }^{16} H L G$, branche VI bis, p. 380-382.

${ }^{17} H L G$, branche IV, p. 258-260.

18 Jean-Charles PAYEN, Le Motif du repentir dans la littérature française médiévale (des origines à 1230), Genève, Droz, 1967, « Le Perlesvaus », p. 419-434, citation p. 423.
} 
scandaleuse. Thomas Kelly, dans Le Haut Livre du Graal: Perlesvaus. A Structural Study ${ }^{19}$, tente de résoudre la difficulté en comparant le roman aux fabliaux, qui prouvent que la mentalité médiévale mêlait brutalité et raffinement. Ce qui paraît normal dans les fabliaux ne devrait pas choquer dans le Perlesvaus, qui serait selon Kelly une œuvre destinée à un public masculin, accordant donc la part du lion à la violence plutôt qu'à la courtoisie. Le texte incarnerait en somme un état d'esprit qui nous est étranger aujourd'hui mais paraissait plus acceptable au public du début $\mathrm{du} \mathrm{XIII}^{\mathrm{e}}$ siècle. Le défaut d'une telle explication est, à bien y regarder, qu'elle fait fi du problème générique : elle éclaire certes le fonctionnement des fabliaux ou des chansons de geste, mais le constat que la mentalité médiévale tolérait plus de «brutalité » que la nôtre ne justifie pas pourquoi cette même brutalité vient se manifester au cœur d'un genre plutôt réputé pour son «raffinement ». Le problème de la violence du Perlesvaus n'est pas qu'il y ait de la violence : c'est qu'il y en ait alors qu'on ne l'attendait pas. Du point de vue du contenu, le Perlesvaus n'est pas beaucoup plus sanglant ou cruel que La Chanson de Roland, mais le contexte générique fait tout, et formate les attentes du lecteur, au Moyen Âge comme aujourd'hui ${ }^{20}$; or le Perlesvaus déjoue manifestement certaines de ces attentes. La justification par le lectorat visé - masculin plutôt que féminin - ne tient pas non plus, puisqu'elle suppose que les autres romans arthuriens avaient un public massivement féminin, ce qui est une supposition difficile à étayer.

\footnotetext{
${ }^{19}$ Thomas E. Kelly, Le Haut Livre du Graal : Perlesvaus. A Structural Study, Genève, Droz, 1974, "The Author and his Public», p. 15-24. Sur les fabliaux, Kelly se réfère principalement aux analyses des Per NyKrog (Les Fabliaux, Copenhague, 1957).

${ }^{20}$ Voir à ce sujet Jean-Marie SCHAEFFER, Qu'est-ce qu'un genre littéraire?, Paris, Éditions du Seuil, 1989. J'emploie l'adjectif «générique » au sens large; dans le cadre de la production narrative médiévale on parlera plus facilement de matiere, mais celle-ci crée exactement le même type d'attentes : le public du XIII ${ }^{\mathrm{e}}$ siècle ne s'attend pas à ce que la matière de Bretagne lui soit contée selon les mêmes modalités que la matière de France.
} 


\section{La cruauté au service de Dieu}

Si le Perlesvaus était simplement un roman arthurien sombre, où la balance penchait plutôt vers la violence que vers la courtoisie, ce serait un texte certes singulier et inhabituel, mais peu problématique dans l'absolu. Or Perlesvaus se proclame «haut livre du Graal», récit éminemment religieux, riche en senefiance et en enseignements. Il est difficile pour le lecteur moderne de concilier violence et quête religieuse ; mais dans le Perlesvaus, plus encore, la violence est religieuse et la religion se fait violente. Le récit met en scène à plusieurs reprises une violence inouïe, justifiée par la foi. Cette violence fondée en religion se manifeste dans deux cas de figure différents.

Le premier de ces cas de figure, qui structure le fonctionnement idéologique du roman, est l'opposition fondamentale entre Ancienne Loi et Nouvelle Loi. L'Ancienne Loi, contre laquelle luttent les chevaliers d'Arthur, est difficile à définir : le nom fait songer au judaïsme, mais les pratiques de cette « religion » ressemblent plutôt à du paganisme (adoration d'idoles, polythéisme, etc.). C'est une sorte de mécréance généralisée et faiblement définie ; face à ses tenants, l'extermination est favorable à la conversion. Après avoir conquis les sectateurs du Taureau de Cuivre, Perlesvaus fait passer les survivants sous l'entrée, défendue par deux automates de cuivre; ceux-ci massacrent les mécréants et épargnent ceux dont la conversion est sincère. Le résultat est sanglant: « de .m. et de .v.c. que il estoient n'en furent gari que .xiii. que il ne fussent tot ocis et ecervelé des maus de fer $»^{21}$. Dieu reconnaîtra les siens, pourrait-on presque dire. On n'est pas très loin d'une «poétique du génocide joyeux », pour reprendre les termes employés par Jean-Charles Payen au sujet du Roland ${ }^{22}$, même si le roman se concentre plus sur le «devoir de violence » que sur le «plaisir de tuer » qui en résulte. Les combats et les massacres n'ont

\footnotetext{
${ }^{21} H L G$, branche IX, p. 664.

${ }^{22}$ Jean-Charles PAYEN, « Une poétique du génocide joyeux : devoir de violence et plaisir de tuer dans la Chanson de Roland», Olifant, 6 (1978-1979), p. 226-236.
} 
rien de jouissif ici : au contraire Perlesvaus est défini par sa peine et les épreuves qu'il traverse.

Nitze $^{23}$ a tenté d'expliquer la grande violence religieuse du Perlesvaus en l'opposant à la Queste, et en affirmant que les deux textes reflétaient des pensées religieuses divergentes, celle de Cluny pour notre roman, celle de Cîteaux pour la Queste. Fanni Bogdanow ${ }^{24}$ a contesté cette interprétation en montrant que l'esprit de croisade qui semble informer la violence du Perlesvaus n'est pas contradictoire avec certains propos de saint Bernard, qui dans le De Laude Novae Militiae ad Milites Templi affirme que «le chrétien se fait gloire de la mort d'un païen, parce que le Christ lui-même s'en est glorifié ${ }^{25}$, et qu'il « vaut mieux de les mettre à mort que de les laisser vivre pour qu'ils portent les mains sur les justes, de peur que les justes, à leur tour, ne se livrent à l'iniquité $»^{26}$. Cette crainte de la contamination traverse tout le roman : le monde chrétien est assiégé par les forces du paganisme, qui entourent le royaume de Logres de toutes parts, isolant la Bretagne au sein d'une mer de perversité. Certains propos du Perlesvaus vont dans le sens des explications de Bogdanow et semblent refléter les deux versants de la pensée de saint Bernard, agressive et contemplative : ainsi la mère de Perlesvaus, dans un long discours, pousse son fils à se venger de ses ennemis, «kar nul preudon ne se doit faire vengier par semblant, mais quanqu'il metroit en chiere et en samblant faire et en manechier, doit il metre en fait quant lieux en vient ${ }^{27}{ }^{27}$; mais tout de suite après elle renonce à sa propre haine envers ses ennemis :

${ }^{23}$ op. cit., II, p. 86-88.

24 Fanni Bogdanow, «Le Perlesvaus », Grundriss der romanischen Literaturen des Mittelalters. Le Roman jusqu'à la fin du XIII ${ }^{e}$ siècle, IV/2, Heidelberg, Carl Winter, 1984, p. $43-67$.

${ }^{25}$ Bernard de Clairvaux, De laude Novae Militiae ad Milites Templi, III, 4, in Cuvres complètes de saint Bernard, Abbé CHARPENTIER (trad.), Paris, 1866, p. 392.

${ }^{26}$ Ibid.

${ }^{27} H L G$, branche VIII, p. 602. 
Je volroie bien que nos anemis fussent tel que il s'amendasent envers nos et que il nos fesissent autretant de bien del nostre, meesme sanz aux grever, con il nos ont fait de mal, par issi conme m'ire et la vostre lor esteroit pardonee. La moie ire lor pardoins jo bien au mien endroit, kar il ne m'est mais mestier que je veille nullui mal ; et Salemons dist que li pechiere qui autrui maldist, maudist soi meesmes. ${ }^{28}$

On assiste à une séparation des fonctions: à Perlesvaus revient la vengeance, à sa mère le pardon; les deux attitudes sont à la fois légitimes et nécessaires. Plus tard, le même héros croisera un spectacle qui le laissera perplexe : au pied d'une croix, deux prêtres se comportent de manière opposée, l'un embrassant et adorant la croix avec de grandes expression de liesse, l'autre la battant à l'aide de verges sans jamais cesser de pleurer ${ }^{29}$. Par la suite un ermite lui expliquera la signification de cet événement : l'attitude de chacun des deux prêtres est correcte, puisque le crucifix est un objet à la fois digne d'amour, parce qu'il symbolise la résurrection du Christ, et de haine, parce qu'il signifie l'ingratitude des hommes envers leur Sauveur ${ }^{30}$. De même, l'économie du Perlesvaus tolère à la fois l'emphase religieuse et l'excès de violence : on peut interpréter ces deux passages - le discours de la mère du héros et la scène avec les deux prêtres - comme des stratégies de légitimation internes à la diégèse.

Le deuxième cas de figure où la violence fonde et se fonde dans un propos religieux est celui de la senefiance, certes moins développée et moins cohérente que celle de la Queste, mais néanmoins présente. Le Perlesvaus présente à plusieurs reprises des scènes apparemment scandaleuses mais dont le sens profond est révélé ensuite par des interprètes, exactement sur le même modèle que la Queste. La scène des deux prêtres face à la croix en est un exemple. Un autre serait le passage célèbre où le roi païen (mais futur chrétien) Gurgaran, à la nouvelle de la mort de son fils, fait bouillir et découper la chair de celui-ci, avant

\footnotetext{
${ }^{28}$ Ibid.

${ }^{29} H L G$, branche IX, p. 626.

${ }^{30} H L G$, branche IX, p. 670-672.
} 
d'en servir les morceaux à tous les habitants de sa cité ${ }^{31}$. Plus tard un prêtre interprétera l'événement pour Gauvain, qui en fut le témoin :

«Sire, jo voil demander d'un roi, fait mesire Gauvain : quant jo li oi son fil aporté mort, il le fist cuire et bolir ; aprés le fist mangier a tos chaus de sa tere. - Sire, fait li provoires, il avoit ja son cuer aporté al Sauveor; si volt tel sacrefise faire de son sanc et de sa char a Nostre Seignor. E por cho en fist il mangier tos chaus de sa tere et volt que lor pensee fust autretele com la soie. Et a si desrachinee sa tere de tote malvaise creance qu'il n'en i a point demoré. $»^{32}$

Au-delà de l'élucidation donnée par le prêtre, le parallèle établi avec l'eucharistie est évident. Mais, comme le remarque Francis Dubost ${ }^{33}$, la violence de la scène excède son explication : on a beau considérer le rituel de Gurgaran comme une allégorie de la conversion ou du sacrifice christique, il n'en demeure pas moins qu'en deçà de tout potentiel allégorique, il s'agit d'une scène réelle d'anthropophagie. La violence résiste à la senefiance et dépasse les bornes qu'elle essaie de lui imposer.

\section{Une violence incompressible}

Ce qui gêne le plus dans le Perlesvaus, c'est sans doute cette capacité de la violence à excéder les limites de l'interprétation: ni les explications stylistiques, ni les justifications idéologiques ou allégoriques ne parviennent à en rendre totalement compte. L'une des scènes les plus cruelles du roman se produit à l'intersection de l'idéologie de la croisade et de l'allégorèse religieuse ; il s'agit du châtiment infligé par Perlesvaus au seigneur des Marais, qui assiégeait le château de sa mère :

\footnotetext{
${ }^{31} H L G$, branche VI, p. 312-314.

${ }^{32} H L G$, branche VI, p. 334.

${ }^{33}$ Francis Dubost, "Le Perlesvaus, livre de haute violence », La Violence dans le monde médiéval, Aix-en-Provence, Presses de l'Université de Provence (Senefiance, 36), 1994, p. 179-199.
} 
Il fait aprester une grant cuve en mi la cort et amener les .xi. chevaliers; il lor fait les chiés couper en la cuve et tant sainier con il peurent rendre de sanc et les cors oster ariere et les chiés, si que il ne n'ot que le sanc tot pur en la cuve ; aprés fait desarmer le Segnor des Mores et amener devant la cuve ou il avoit grant fuison de sanc. Il li fait les mains lier et les piez molt estroit. Aprés li dist : " Sire des Mores, vos ne peustes onques estre saoulez del sanc as chevaliers ma dame ma mere, mais ge vos saolerai del sanc as vostres ! » Il le fait pendre en la cuve par les piés, si que la teste fu el sanc dusque as espaules, puis le fait tant tenir que il fu noez et estainz. Aprés fait porter son cors et les autres et toz les chiés as chevaliers en un charnier anchien qui iert dejoste une viés chapele en la forest, et le cuve a tot le sanc, fist jeter en la riviere, si que li aigue fu tote sanglente. ${ }^{34}$

On peut interpréter ce châtiment à la lumière de l'opposition Ancienne Loi/Nouvelle Loi, et décréter que le seigneur des Marais n'a que ce qu'il mérite : à l'instar des «méchants » dans les films hollywoodiens à grand spectacle, les païens du Perlesvaus ne sont pas là pour nous inspirer de la pitié. Méritent-ils pour autant d'être noyés dans une cuve remplie du sang de leurs propres guerriers ? Le versant guerrier de l'éthique cistercienne ne suffit pas à le justifier. C'est à ce stade qu'intervient l'explication allégorique : le seigneur des Marais meurt par là où il a péché - qui vit par le sang périt par le sang. La punition inventée par Perlesvaus est une sorte de contrappasso dantesque avant la lettre, une adéquation parfaite du châtiment au crime ${ }^{35}$. Mais on se retrouve dans le même cas de figure que l'anthropophagie de Gurgaran: la violence «allégorique » est plus violente qu'allégorique, et Perlesvaus, le noble héros du roman, commet un acte qui, tout en ayant une portée symbolique forte, demeure à nos yeux cruel et barbare.

\footnotetext{
${ }^{34}$ HLG, branche VIII, p. 612-614.

${ }^{35}$ D'ailleurs, on retrouve presque le même supplice dans La Divine comédie: les violents contre leurs prochains sont plongés dans le Phlégéton, fleuve de sang bouillant, la seule différence étant qu'ils ont la tête en haut et non en bas. Voir DanTE, Euvres complètes, Christian BEC (dir.), Paris, Pochothèque, 1996, La Divine comédie, Enfer, XII, v. 100 sqq., p. 645-647.
} 
Le Perlesvaus contient un degré de violence incompressible que les analyses idéologiques ne parviennent pas à justifier. Anne Berthelot a tenté une explication psychanalytique : «Le Perlesvaus constitue le triomphe du refoulé, admis par défaut sur la scène textuelle à partir d'un raisonnement syllogistique, et supplantant le discours théologique par la force/violence brute de ses images, infiniment plus cohérentes et lancinantes que les arguties rhétoriques de l'allégorèse $»^{36}$. Le système allégorique (peut-être en raison de son manque de cohérence, à l'opposé de la Queste) peine à étreindre tout ce qu'il génère, et révèle ce qui devait rester caché : l'inconscient d'un auteur (on retrouverait dans ce cas les commentaires de Loomis sur l'idiosyncrasie pathologique du rédacteur du roman), ou de toute une société (et dans ce cas le Perlesvaus constituerait un document psychanalytique sur l'époque de sa composition). Francis Dubost, dans son article déjà cité, se rapproche de cette interprétation. Pour lui le Perlesvaus veut à tout prix relancer l'esprit de croisade à une époque où il s'éteint; mais en sous-main, le roman exprime aussi (et surtout) « une volonté [cistercienne] farouche de fermer les yeux sur le monde terrestre, acharnement pathétique à ignorer le corps, à ignorer la souffrance, à faire violence à la chair, afin de transposer les réalités sensibles dans l'ordre des valeurs spirituelles, où beauté et violence, devenues de purs concepts, cessent d'affecter les sens. ${ }^{37}$ L'exubérante violence du Perlesvaus exprime moins une affirmation qu'un refus : elle révèle par des moyens détournés un idéal ascétique, ou plutôt sa face cachée, ce qu'il doit refouler pour être lui-même.

Charles Méla dans La Reine et le Graal appelait déjà le Perlesvaus un « roman fantasmagorique », et les propos d'Anne Berthelot et de Francis Dubost semblent aller dans ce sens. Peut-être faudrait-il, en fin de compte, interpréter le

\footnotetext{
${ }^{36}$ Anne Berthelot, "Violence et Passion, ou la christianisme sauvage de Perlesvaus : le Haut Livre du Graal », La Violence dans le monde médiéval, Aix-en-Provence, Presses de l’Université de Provence (Senefiance, 36), 1994, p. 19-36 ; citation p. 35.

${ }^{37}$ art. cit., p. 199.
} 
Perlesvaus d'une façon qui unirait le fantasmagorique et le religieux. L'univers dans lequel se déroule l'action du roman n'est pas le monde «normal» : les lois qui régissent la vie de tous les jours - épargner les adversaires qui demandent grâce, faire preuve de mesure dans la rétribution, éviter l'excès de violence - ne s'appliquent pas. Le sens allégorique prend le pas sur la bienséance, les châtiments n'offrent aucune place à la repentance mais visent à l'exemplarité, les actes les plus inouïs sont justifiés par la senefiance qu'ils révèlent. Thomas Kelly remarquait que l'eschatologie assumait un rôle important dans la dernière partie du roman, lorsque la guerre menace d'engloutir le royaume d'Arthur et que Perlesvaus vit ses dernières aventures ${ }^{38}$. Mais avec le supplice du seigneur des Marais, allégorie des souffrances infernales, n'est-on pas déjà dans une logique eschatologique ? Sans doute ce filtre peut-il être étendu à l'ensemble du roman : le Perlesvaus serait dans ce cas un récit se déroulant dans une logique de fin des temps, de jugement dernier, où le pardon et la merci n'ont plus lieu d'être. La bienséance et la clémence sont des valeurs hypocrites dans un univers en proie à l'urgence : voilà peut-être ce qui explique les apparentes aberrations, ainsi que l'extrême singularité du roman dans le champ de la littérature arthurienne en prose. Malgré les apparences, le Perlesvaus ne se déroule peutêtre pas dans la Bretagne du début de l'ère chrétienne, mais dans un monde spectaculaire où chaque décision est finale, où le Bien et le Mal sont d'une évidence aveuglante, et où rien ne peut empêcher l'inexorable accomplissement de la Vérité.

${ }^{38}$ op. cit., p. 106 sqq. 